\title{
The use of a Riesz fractional differential-based approach for texture enhancement in image processing
}
Q. $\mathrm{Yu}^{1}$
F. $\mathrm{Liu}^{2}$
I. Turner ${ }^{3}$
V. $\operatorname{Vegh}^{5}$
K. Burrage ${ }^{4}$

(Received 31 October 2012; revised 13 August 2013)

\begin{abstract}
Texture enhancement is an important component of image processing that finds extensive application in science and engineering. The quality of medical images, quantified using the imaging texture, plays a significant role in the routine diagnosis performed by medical practitioners. Most image texture enhancement is performed using classical integral order differential mask operators. Recently, first order fractional differential operators were used to enhance images. Experimentation with these methods led to the conclusion that fractional differential operators not only maintain the low frequency contour
\end{abstract}

http://journal.austms.org.au/ojs/index.php/ANZIAMJ/article/view/6325 gives this article, (c) Austral. Mathematical Soc. 2013. Published September 25, 2013, as part of the Proceedings of the 16th Biennial Computational Techniques and Applications Conference. ISSN 1446-8735. (Print two pages per sheet of paper.) Copies of this article must not be made otherwise available on the internet; instead link directly to this URL for this article. 
features in the smooth areas of the image, but they also nonlinearly enhance edges and textures corresponding to high frequency image components. However, whilst these methods perform well in particular cases, they are not routinely useful across all applications. To this end, we apply the second order Riesz fractional differential operator to improve upon existing approaches of texture enhancement. Compared with the classical integral order differential mask operators and other first order fractional differential operators, we find that our new algorithms provide higher signal to noise values and superior image quality.

Subject class: 26A33, 92C55

Keywords: Fractional calculus, texture enhancement, image processing

\section{Contents}

1 Introduction

C591

2 The improved fractional differential mask

C593

3 Experiments and analysis

C598

4 Conclusions

C602

References

C605

\section{Introduction}

Texture enhancement is one of the most important issues in image processing and plays a substantial role in medical imaging [1]. The quality of images, especially the texture, is increasingly significant for clinical diagnosis of pathology. 
Current image enhancement algorithms are typically based on integral order differential mask operators that include the Sobel, Roberts, Prewit and Laplacian techniques [1, 2]. After a comparison of images enhanced using first and second order derivatives, it was concluded that the integral order differential operator has several shortcomings. In particular, processing using first order masks produces wide edges, while second order masks are sensitive to noise and generate double responses when the grey scale changes [2, 3].

Recently, the advantages of fractional calculus were widely demonstrated for various applications in science and engineering [4, 5]. These successes motivated researchers to apply fractional derivatives to digital image processing $[3,6,7,8,9,10,11]$. Zhang et al. [3] developed an algorithm based on the Riemann-Liouville definition and applied the resulting model with a fractional derivative index between one and two to enhance the texture and edges of a digital image. Sejdić et al. [6] investigated the use of the fractional Fourier transform in signal processing. Pesquet-Popescu and Véhel [7] developed stochastic fractal models for image processing. Mathieu et al. [8] applied fractional differentiation for edge detection. Gao et al. [9] applied a quaternion fractional differential based on the Grünwald-Letnikov definition to a colour image. Gao et al. [10] applied an improved fractional differential operator based on a piecewise quaternion for image enhancement. Pu et al. [11] proposed various algorithms based on the Grünwald-Letnikov definition which work well for grey images but produce colour images which are distorted in the RGB (red, green and blue) space.

In contrast to the standard derivative, the fractional derivative of a direct current or low frequency signal is usually nonzero $[3,9,11]$. Thus, the fractional differential not only nonlinearly preserves the contour features of the smooth area, but maintains high frequency edge features in those areas where the grey changes considerably. It also preserves high frequency texture detailed features in those areas where the grey does not change significantly.

We build upon the classical and first order fractional mask approaches to overcome defects in enhancement and colour image distortion by investigat- 
ing the performance of two fractional differential algorithms based on the Riesz fractional differential operator [5]. In Section 2 we present the theoretical analysis for implementing fractional differential masks and define two fractional derivative algorithms, FCD-1 and FCD-2, based on the Riesz fractional differential operator. Finally, we illustrate that our algorithms provide higher precision and better visual effects for texture enhancement than Pu's algorithm (YIFEIPU-1) [11] when applied to medical imaging applications.

\section{The improved fractional differential mask}

The $v$ order $(0<v \leqslant 2)$ Riesz fractional derivative $\partial^{v} u(t) / \partial|t|^{v}$ for the infinite interval $-\infty<\mathrm{t}<+\infty$ is [5]

$$
\frac{\partial^{v} u(t)}{\partial|t|^{v}}=-c_{v}\left(\frac{\partial^{v}}{\partial t^{v}}+\frac{\partial^{v}}{\partial(-t)^{v}}\right) u(t),
$$

where $c_{v}=[2 \cos (\pi v / 2)]^{-1}$ with $v \neq 1, p-1<v \leqslant p \leqslant 2$ for $p \in \mathbb{N}$, and

$$
\begin{aligned}
\frac{\partial^{v} u(t)}{\partial t^{v}} & =\frac{1}{\Gamma(p-v)} \frac{\partial^{p}}{\partial t^{p}} \int_{-\infty}^{t} \frac{u(\xi) d \xi}{(t-\xi)^{v+1-p}} \\
\frac{\partial^{v} u(t)}{\partial(-t)^{v}} & =\frac{(-1)^{p}}{\Gamma(p-v)} \frac{\partial^{p}}{\partial t^{p}} \int_{t}^{+\infty} \frac{u(\xi) d \xi}{(\xi-t)^{v+1-p}} .
\end{aligned}
$$

The Riesz fractional derivative is approximated using the second order fractional centered difference scheme given by Ortigueira [12] with step $h$,

$$
\frac{\partial^{v} \mathrm{u}(\mathrm{t})}{\partial|\mathrm{t}|^{v}}=-\frac{1}{\mathrm{~h}^{v}} \sum_{\mathrm{k}=-\infty}^{\infty} \frac{(-1)^{\mathrm{k}} \Gamma(v+1)}{\Gamma\left(\frac{v}{2}-\mathrm{k}+1\right) \Gamma\left(\frac{v}{2}+\mathrm{k}+1\right)} \mathrm{u}(\mathrm{t}-\mathrm{kh})+\mathrm{O}\left(\mathrm{h}^{2}\right) .
$$

We define two fractional differential algorithms, FCD-1 and FCD-2, based on the Riesz fractional differential operator (1). Firstly, when $0<v<1$, (4) is 
rewritten as

$$
\begin{aligned}
\frac{\partial^{v} u(t)}{\partial|t|^{v}} & =-\frac{1}{2 \cos (\pi v / 2)}\left(\frac{\partial^{v}}{\partial t^{v}}+\frac{\partial^{v}}{\partial(-t)^{v}}\right) u(t) \\
& \approx-\frac{1}{2 \cos (\pi v / 2) h^{v}}\left[\sum_{k=0}^{\infty} \omega_{k} u(t-k h)+\sum_{k=-\infty}^{0} \omega_{k} u(t-k h)\right]
\end{aligned}
$$

where

$$
\begin{aligned}
& \omega_{0}=-\frac{\Gamma(1-v / 2)}{v \Gamma(1+v / 2) \Gamma(-v)} \\
& \omega_{k}=\frac{(-1)^{k+1} \Gamma(v / 2) \Gamma(1-v / 2)}{\Gamma(v / 2-k+1) \Gamma(v / 2+k+1) \Gamma(-v)} \quad \text { for } k= \pm 1, \pm 2, \ldots
\end{aligned}
$$

In the context of medical images, Zhang et al. [3] and Pu et al. [11] discuss the biggest variable of the grey level being limited, and the shortest distance for a change in the grey level image must be at an adjacent pixel. Thus, pixels are used to measure the duration of a two dimensional digital image $s(x, y)$ with respect to two variables $x$ and $y$. As an example, for a two dimensional digital image $s(x, y)$ at pixel $\left(x^{*}, y^{*}\right)$, on the positive $x$-axis within the region $\left[0, x^{*}\right]$, the $N+1$ pixels are $s_{N}\left(x^{*}, y^{*}\right)=s\left(0, y^{*}\right), \ldots, s_{k}\left(x^{*}, y^{*}\right)=s\left(x^{*}-k h, y^{*}\right)$, $\ldots, s_{0}\left(x^{*}, y^{*}\right)=s\left(x^{*}, y^{*}\right)$, where $h=x^{*} / N$, and $N$ is the number of divisions. Similar results hold for the other directions, such as negative $x$-axis, negative $y$ axis, positive $y$-axis and so on. Generally, we assume that $h=1$. After truncation, the anterior $n+1$ approximate fractional centered difference of the fractional partial differential on the positive $\boldsymbol{x}$-axis is

$$
\frac{\partial^{v} s(x, y)}{\partial x^{v}} \cong-\frac{1}{2 \cos (\pi v / 2) h^{v}} \sum_{k=0}^{n} \omega_{k} s(x-k h, y), \quad 0<v<1
$$

We implemented fractional derivative masks along eight symmetric directions, the negative $x$-axis, positive $x$-axis, negative $y$-axis, positive $y$-axis, left downward diagonal, right upward diagonal, left upward diagonal, and right 
(a)

\begin{tabular}{|c|c|c|c|c|c|c|}
\hline & $\cdots$ & 0 & $C_{s_{n}}$ & 0 & $\cdots$ & \\
\hline & $\cdots$ & 0 & $C_{s_{n-1}}$ & 0 & $\cdots$ & \\
\hline & & $\vdots$ & $\vdots$ & $\vdots$ & & \\
\hline & $\cdots$ & 0 & $C_{s_{k}}$ & 0 & $\cdots$ & \\
\hline & & $\vdots$ & $\vdots$ & $\vdots$ & & \\
\hline & $\cdots$ & 0 & $C_{s_{1}}$ & 0 & $\cdots$ & \\
\hline & $\cdots$ & 0 & $C_{s_{0}}$ & 0 & $\cdots$ & \\
\hline
\end{tabular}

(b)

\begin{tabular}{|c|c|c|c|c|c|c|}
\hline & $\cdots$ & 0 & $\mathrm{C}_{s_{0}}$ & 0 & $\cdots$ & \\
\hline$\cdots$ & 0 & $\mathrm{C}_{s_{1}}$ & 0 & $\cdots$ & \\
\hline & & $\vdots$ & $\vdots$ & $\vdots$ & & \\
\hline$\cdots$ & 0 & $\mathrm{C}_{s_{k}}$ & 0 & $\cdots$ & \\
\hline & & $\vdots$ & $\vdots$ & $\vdots$ & & \\
\hline$\cdots$ & 0 & $\mathrm{C}_{s_{n-1}}$ & 0 & $\cdots$ & \\
\hline & $\cdots$ & 0 & $\mathrm{C}_{s_{n}}$ & 0 & $\cdots$ & \\
\hline
\end{tabular}

(c)

\begin{tabular}{|c|c|c|c|c|c|c|}
\hline & & & & & & \\
\hline$\vdots$ & $\vdots$ & & $\vdots$ & & $\vdots$ & $\vdots$ \\
\hline 0 & 0 & $\cdots$ & 0 & $\cdots$ & 0 & 0 \\
\hline$C_{s_{n}}$ & $C_{s_{n-1}}$ & $\cdots$ & $C_{s_{k}}$ & $\cdots$ & $C_{s_{1}}$ & $C_{s_{0}}$ \\
\hline 0 & 0 & $\cdots$ & 0 & $\cdots$ & 0 & 0 \\
\hline$\vdots$ & $\vdots$ & & $\vdots$ & & $\vdots$ & $\vdots$ \\
\hline & & & & & & \\
\hline
\end{tabular}

(d)

\begin{tabular}{|c|c|c|c|c|c|c|}
\hline & & & & & & \\
\hline$\vdots$ & $\vdots$ & & $\vdots$ & & $\vdots$ & $\vdots$ \\
\hline 0 & 0 & $\cdots$ & 0 & $\cdots$ & 0 & 0 \\
\hline $\mathrm{C}_{\mathrm{s}_{0}}$ & $\mathrm{C}_{\mathrm{s}_{1}}$ & $\cdots$ & $\mathrm{C}_{\mathrm{s}_{\mathrm{k}}}$ & $\cdots$ & $\mathrm{C}_{\mathrm{s}_{\mathrm{n}-1}}$ & $\mathrm{C}_{\mathrm{s}_{\mathrm{n}}}$ \\
\hline 0 & 0 & $\cdots$ & 0 & $\cdots$ & 0 & 0 \\
\hline$\vdots$ & $\vdots$ & & $\vdots$ & & $\vdots$ & $\vdots$ \\
\hline & & & & & & \\
\hline
\end{tabular}

Figure 1: Fractional differential mask for four of the eight directions: (a) $W_{1}^{*}$, negative $x$-axis; (b) $W_{2}^{*}$, positive $x$-axis; (c) $W_{3}^{*}$, negative $y$-axis; (d) $W_{4}^{*}$, positive $y$-axis.

downward diagonal, respectively denoted by $W_{l}^{*}$ for $l=1,2, \ldots, 8$ (see Figures 1 and 2). These directions ensure that the fractional differential masks have an anti-rotation capability. The masks are $W_{l}^{*}=W_{l}(i, j)$ where $M_{l} \leqslant i \leqslant N_{l}, P_{l} \leqslant j \leqslant Q_{l}$, and the details of $M_{l}, N_{l}, P_{l}$ and $Q_{l}$ for $l=1,2, \ldots, 8$ are shown after $(9)$.

In Figures 1 and 2, $\mathrm{C}_{s_{0}}$ is the mask coefficient associated with the pixel of interest. In general, to ensure that the fractional differential mask has a certain center, $n$ is chosen as an even number. When $n=2 m$ is an even number, one implements a $(2 m+1) \times(2 m+1)$ fractional differential mask. 
(a)

\begin{tabular}{|c|c|c|c|c|c|c|}
\hline & & & & $\cdots$ & 0 & $C_{s_{0}}$ \\
\hline & & & $\cdots$ & 0 & $C_{s_{1}}$ & 0 \\
\hline & & &.$\cdot$ & $\cdot$ & 0 & $\vdots$ \\
\hline & $\cdots$ & 0 & $C_{s_{k}}$ & 0 & $\cdots$ & \\
\hline$\vdots$ & 0 &.$\cdot$ &.$\cdot$ & & & \\
\hline 0 & $C_{s_{n-1}}$ & 0 & $\cdots$ & & & \\
\hline$C_{s_{n}}$ & 0 & $\cdots$ & & & & \\
\hline
\end{tabular}

(c)

\begin{tabular}{|c|c|c|c|c|c|c|}
\hline $\mathrm{C}_{s_{n}}$ & 0 & $\cdots$ & & & & \\
\hline 0 & $\mathrm{C}_{s_{n-1}}$ & 0 & $\cdots$ & & & \\
\hline$\vdots$ & 0 & $\ddots$ & $\ddots$ & & & \\
\hline & $\cdots$ & 0 & $C_{s_{k}}$ & 0 & $\cdots$ & \\
\hline & & & $\ddots$ & $\ddots$ & 0 & $\vdots$ \\
\hline & & & $\cdots$ & 0 & $C_{s_{1}}$ & 0 \\
\hline & & & & $\cdots$ & 0 & $C_{s_{0}}$ \\
\hline
\end{tabular}

(d)

\begin{tabular}{|c|c|c|c|c|c|c|}
\hline $\mathrm{C}_{\mathrm{s}_{0}}$ & 0 & $\cdots$ & & & & \\
\hline 0 & $\mathrm{C}_{\mathrm{s}_{1}}$ & 0 & $\cdots$ & & & \\
\hline$\vdots$ & 0 & $\ddots$ & $\ddots$ & & & \\
\hline & $\cdots$ & 0 & $\mathrm{C}_{\mathrm{s}_{\mathrm{k}}}$ & 0 & $\cdots$ & \\
\hline & & & $\ddots$ & $\ddots$ & 0 & $\vdots$ \\
\hline & & & $\cdots$ & 0 & $\mathrm{C}_{\mathrm{s}_{\mathfrak{n}-1}}$ & 0 \\
\hline & & & & $\cdots$ & 0 & $\mathrm{C}_{\mathrm{s}_{\mathrm{n}}}$ \\
\hline
\end{tabular}

Figure 2: Fractional differential mask for four of the eight directions: (a) $W_{5}^{*}$, left downward diagonal; (b) $W_{6}^{*}$, right upward diagonal; (c) $W_{7}^{*}$, left upward diagonal; (d) $W_{8}^{*}$, right downward diagonal.

Digital image processing is based on direct processing for discrete pixels, and the algorithm also describes an airspace filtering scheme [11]. The principle of the airspace filter is to move the mask pixel by pixel. There are considerable differences in the properties of grey images and colour images. Thus, there are two algorithms for the fractional differential mask, one for grey images and the other for colour images. To treat an $\mathrm{N}_{x} \times \mathrm{N}_{y}$ digital grey image $s(x, y)$, where $\mathrm{N}_{x}$ and $\mathrm{N}_{y}$ are the dimensions of the image matrix, we perform a convolution filter on the eight symmetric directions with a $(2 m+1) \times(2 m+1)$ mask, and proposed that the fractional differential mask on the eight directions be 
computed using

$$
\begin{aligned}
s_{l}(x, y)= & \sum_{i=M_{l}}^{N_{l}} \sum_{j=P_{l}}^{Q_{l}} W_{l}(i, j) s(x+i, y+j) \quad \text { for } l=1,2,3,4, \\
s_{l}(x, y)= & \sum_{i=M_{l}}^{N_{l}} \sum_{j=P_{l}}^{Q_{l}} 2^{-v / 2} W_{l}(i, j) s(x+i, y+j) \\
& +\left(1-2^{-v / 2}\right) W_{l}(0,0) s(x, y) \quad \text { for } l=5,6,7,8 .
\end{aligned}
$$

where
$M_{1}=-2 m$,
$\mathrm{N}_{1}=0$,
$P_{1}=-m$,
$\mathrm{Q}_{1}=\mathrm{m}$
$\mathrm{M}_{2}=0$,
$\mathrm{N}_{2}=2 \mathrm{~m}$,
$\mathrm{P}_{2}=-\mathrm{m}$,
$\mathrm{Q}_{2}=\mathrm{m}$;
$M_{3}=-m$,
$\mathrm{N}_{3}=\mathrm{m}$,
$P_{3}=-2 m$,
$\mathrm{Q}_{3}=0$;
$\mathrm{M}_{4}=-\mathrm{m}$,
$\mathrm{N}_{4}=\mathrm{m}$,
$\mathrm{P}_{4}=0$,
$\mathrm{Q}_{4}=2 \mathrm{~m}$;
$\mathrm{M}_{5}=0$,
$\mathrm{N}_{5}=2 \mathrm{~m}$,
$P_{5}=-2 m$,
$\mathrm{Q}_{5}=0$;
$\mathrm{M}_{6}=-2 \mathrm{~m}$,
$\mathrm{N}_{6}=0$,
$\mathrm{P}_{6}=0$,
$\mathrm{Q}_{6}=2 \mathrm{~m}$;
$M_{7}=-2 m$,
$\mathrm{N}_{7}=0$,
$P_{7}=-2 m$,
$\mathrm{Q}_{7}=0 ;$
$M_{8}=0$,
$\mathrm{N}_{8}=2 \mathrm{~m}$,
$\mathrm{P}_{8}=0$,
$\mathrm{Q}_{8}=2 \mathrm{~m}$.

Thus,

$$
s(x, y)=\frac{\sum_{l=1}^{8} s_{l}(x, y)}{4\left[\sum_{k=0}^{n} C_{s_{k}}+\sum_{k=1}^{n} 2^{-v / 2} C_{s_{k}}+C_{s_{0}}\right]},
$$

where $C_{s_{k}}$ is the mask coefficient (see (11) and (12)). For a digital RGB colour image the algorithm is similar to that for a grey image, but the fractional differential is applied individually to each of the R, G and B components.

When $0<v<1$ we implement the fractional mask on the eight symmetric directions using what we call the FCD-1 operator, which has the same structure 
as YIFEIPU-1 but with different coefficients. The mask coefficients of the FCD-1 operator are

$$
C_{s_{k}}=-\frac{1}{2 \cos (\pi v / 2) h^{v}} \omega_{k} \quad \text { for } k=0,1,2, \ldots, n,
$$

which ensures that the fractional differential operator FCD-1 produces a sparse matrix of dimension $n+1$. Moreover, all the coefficients depend on the fractional differential order $v$. It can be proven that the sum of the coefficients is nonzero, which is a remarkable difference between the fractional differential mask and the integral differential mask.

Similarly, when $1<v<2$, we define the fractional mask FCD-2 with the coefficients

$$
\begin{aligned}
C_{s_{0}} & =\frac{1}{2 \cos (\pi v / 2) h^{v}} \frac{(v-1) \Gamma(1-v / 2)}{\Gamma(1+v / 2) \Gamma(2-v)}, \\
C_{s_{k}} & =\frac{1}{2 \cos (\pi v / 2) h^{v}} \frac{(-1)^{k} v(v-1) \Gamma(v / 2) \Gamma(1-v / 2)}{\Gamma(v / 2-k+1) \Gamma(v / 2+k+1) \Gamma(2-v)},
\end{aligned}
$$

for $k=1,2, \ldots, n$.

\section{Experiments and analysis}

In this section, we apply our algorithms to image enhancement of grey and colour images. We also compare our methods with YIFEIPU-1 to show that they have better performance in terms of precision and give an improved visual effect for texture enhancement. We adopt the fractional derivative with index $0<v<1$ to enhance the texture details, and use the index $1<v<2$ to enhance the edge and texture details for digital image processing. For the simulations performed here, all algorithms are written and executed using Matlab. For example, Algorithm 1 is for a grey image. 
Algorithm 1: Algorithm for grey image

Input: Read original grey image and add Gaussian noise with mean 0 and variance 0.01 .

Output: $s(x, y)$

1 Choose $\mathrm{m}=2, \mathrm{n}=4$ and mask $5 \times 5$;

2 Compute the mask coefficients $\mathrm{C}_{s_{\mathrm{k}}}$ using either equation (11) $(0<v<1)$ or $(12)(1<v<2)$;

3 Compute the dimension $\mathrm{N}_{x} \times \mathrm{N}_{y}$ of the image matrix;

4 for $x=2: \mathrm{N}_{\mathrm{x}}-1 \mathrm{do}$

5 for $y=2: \mathrm{N}_{\mathrm{y}}-1 \mathrm{do}$

$6 \quad$ for $l=1: 8 \mathrm{do}$

$7 \quad \mid$ Compute $s_{l}(x, y)$ using equations (8) and (9);

$8 \quad$ end

$9 \quad$ Compute $s(x, y)$ using equation (10);

10

11 end

end

12 Display adjusted image $s(x, y)$.

In Figure 3 we compare our method FCD- 1 with YIFEIPU- 1 and the traditional integral differential methods involving the Sobel operator and Laplacian operator on the Lena image [2] with Gaussian noise with mean 0 and variance 0.01. Figure 3 shows that integral differential methods cannot be used for texture enhancement because they enhance high frequency marginal information where the grey level changes greatly but cannot preserve the low frequency contour information in smooth areas. They also cannot enhance the texture details in those areas where grey levels have changed little. We also see that the image obtained from our FCD-1 method has the best definition and the best visual effect. The visual effect obtained by FCD-1 is better than that of YIFEIPU-1 when using the same fractional order and same mask dimensions. We define the signal to noise ratio

$$
\operatorname{SNR}=\left(A_{\text {signal }} / A_{\text {noise }}\right)^{2},
$$


(a)

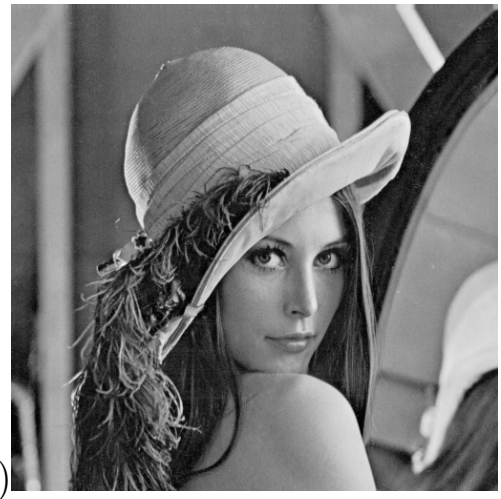

(b)

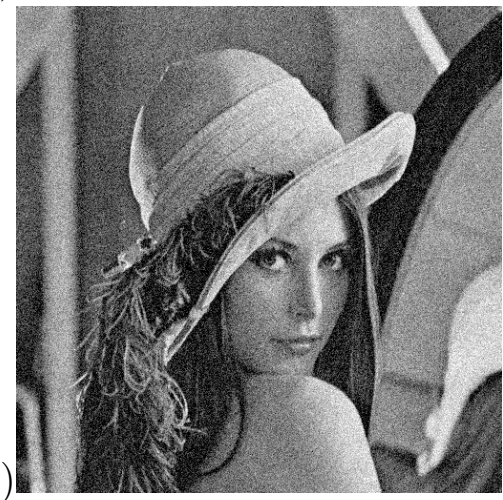

(c)

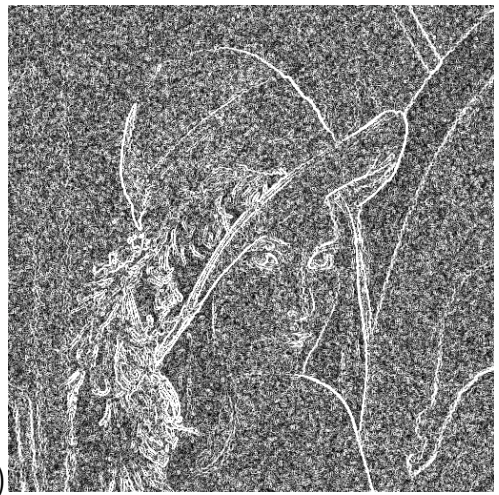

(d)

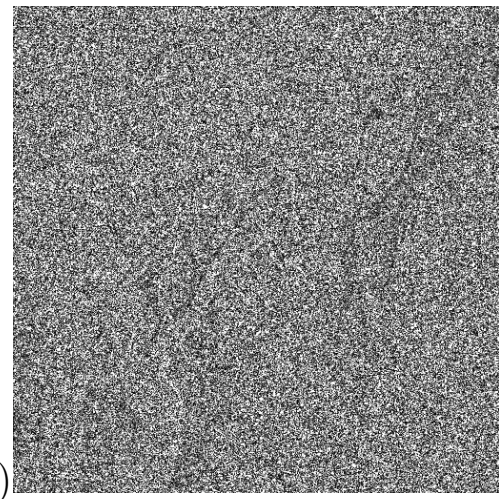

(e)

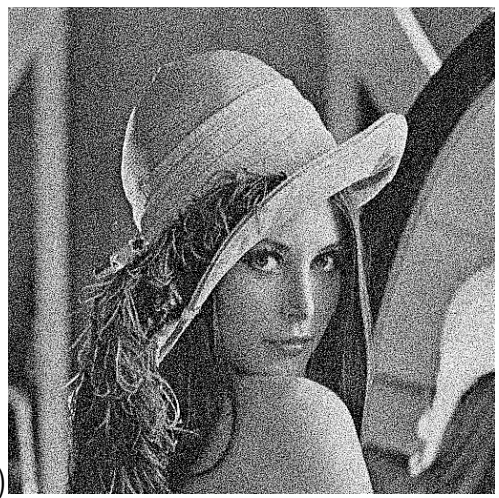

(f)

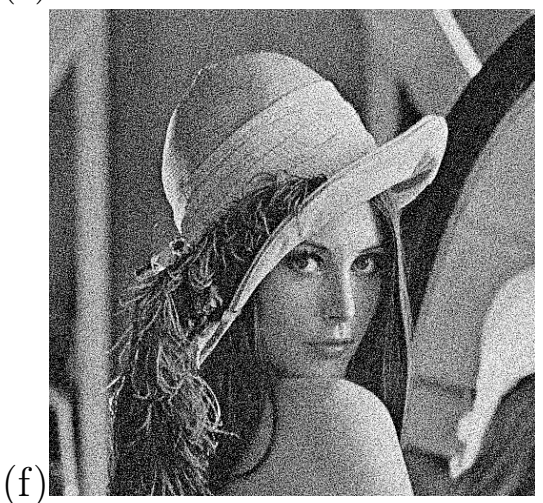

Figure 3: The contrast effects of Gaussian noise image enhancement with mean 0 and variance 0.01 and its fractional differential using YIFEIPU- 1 and FCD-1. (a) Original Lena image, (b) grey scale noise image, (c) Sobel operator, (d) Laplacian operator, (e) 0.5 order YIFEIPU-1 with mask $3 \times 3$, (f) 0.5 order FCD-1 with mask $3 \times 3$. 
Table 1: SNR with Gaussian noise with mean 0 and variance 0.01 with $v=0.5$ and mask $5 \times 5$ for YIFEIPU- 1 and FCD- 1

\begin{tabular}{lc} 
Operators & SNR \\
\hline Sobel & 3.5 \\
Laplacian & 3.3 \\
YIFEIPU-1 & 8.3 \\
FCD-1 & 10.2
\end{tabular}

Table 2: SNR with Gaussian noise with mean 0 and variance 0.01 for both FCD-1 and YIFEIPU- 1 with $v=0.5$

\begin{tabular}{ccc} 
Mask dimensions & YIFEIPU- 1 & FCD- 1 \\
\hline $3 \times 3$ & 10.7 & 13.3 \\
$5 \times 5$ & 8.3 & 10.2 \\
$7 \times 7$ & 7.3 & 8.8 \\
$9 \times 9$ & 6.8 & 8.0 \\
$11 \times 11$ & 6.4 & 7.5
\end{tabular}

where $A$ is root mean square amplitude. Table 1 exhibits the SNR for the traditional integral differential methods using Sobel and Laplacian operators on the Lena image, and we choose $v=0.5$ and mask $5 \times 5$ for YIFEIPU- 1 and FCD-1. Table 1 shows that FCD-1 has a higher SNR than the YIFEIPU-1, Sobel and Laplacian operators, which implies a superior texture enhancement.

For the index $v=0.5$, Table 2 shows a comparison of SNR for the Lena image generated using FCD-1 and YIFEIPU-1. Table 2 shows again that, with the same mask dimensions, FCD-1 has the higher SNR.

We now present two experiments to test our methods on a human brain image from a patient, diagnosed with Parkinson's disease, before surgery from St Andrew's War Memorial Hospital, Brisbane, Australia. 
By individually applying the fractional differential to the three elements in HSI colour space, and then reverting to RGB colour space, one gains a colour image without distortion. Figure 4 compares the texture details of an original fractional anisotropy weighted orientation map and its fractional differential using FCD- 1 with a $5 \times 5$ mask. In Figure 4 the background of the original image is those areas with smooth textures and the most comprehensive texture details; moreover, the grey levels have little variation. The foreground of the original image is those areas with high frequency verge, and grey levels have more variability.

Figure 5 compares texture segmentation performance of an original grey image and its fractional differential version using FCD-2 with a $5 \times 5$ mask. Figure 5 demonstrates that the fractional differential with $1<v<2$ is able to segment texture, as defined by image contrast. As the order increases, the tissue sharpening effect is more pronounced.

\section{Conclusions}

The application of fractional differentials to the analysis and processing of image signals, in particular those associated with digital images, has recently attracted considerable attention.

We derive two fractional differential algorithms for grey and colour image enhancement, FCD-1 and FCD-2, based on the Riesz fractional differential operator. The experiments show that our algorithms are able to produce better results than traditional integral differential based algorithms and fractional methods based on the Grünwald-Letnikov definition. This is the case where nonlinear enhancement of high frequency edges and texture information does not change significantly. We conjecture that the use of a symmetric second order Riesz fractional operator, instead of a one sided first order fractional Grünwald-Letnikov operator, will provide better texture enhancement of the image, and this is confirmed in our results. Furthermore, we performed a 
(a)

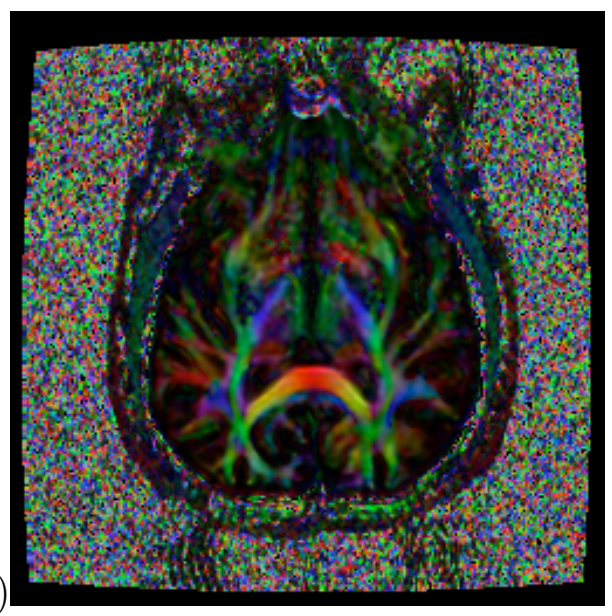

(c)

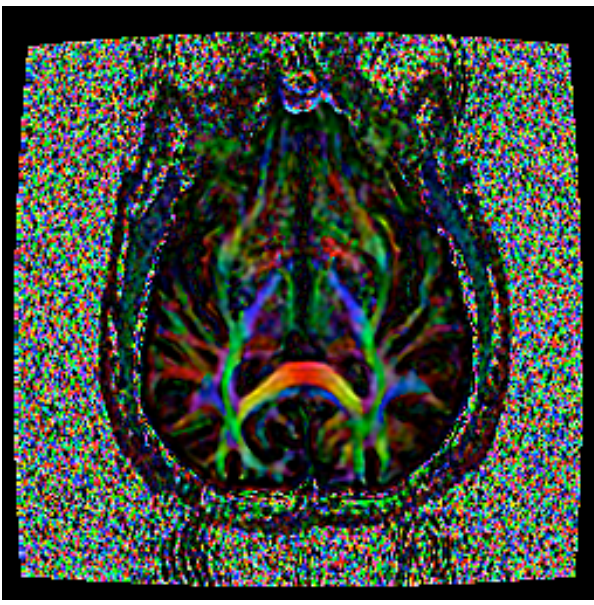

(b)
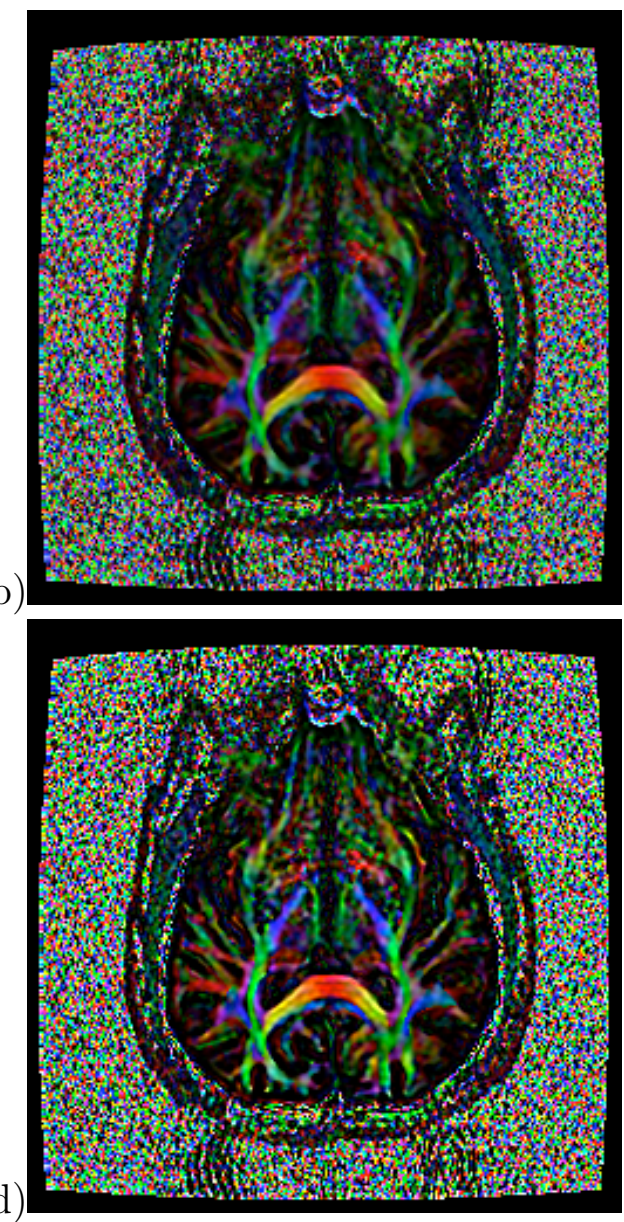

Figure 4: Comparison of texture details between an original fractional anisotropy weighted orientation map and its fractional differential using FCD-1 with a $5 \times 5$ mask. (a) Original image, (b) 0.3 order FCD-1, (c) 0.5 order FCD-1, (d) 0.7 order FCD-1. 
(a)

(b)

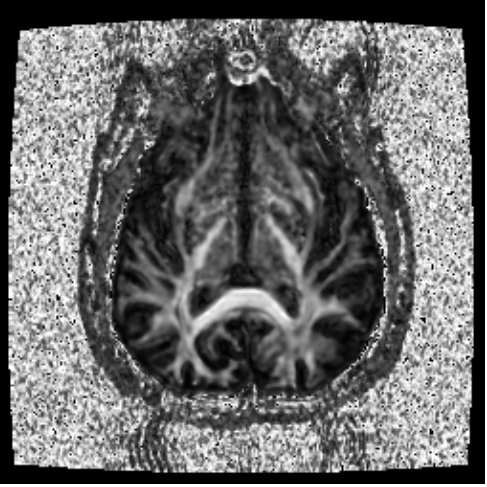

(d)

(e)

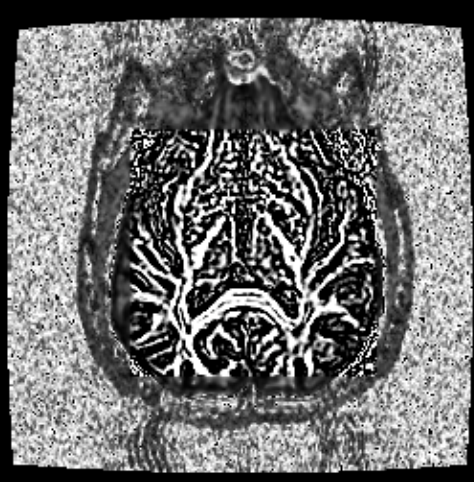

(c)

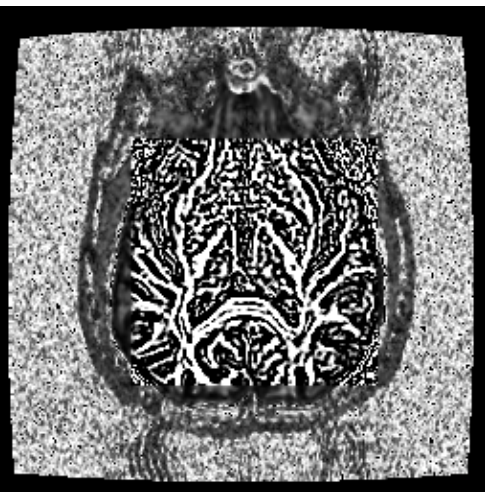

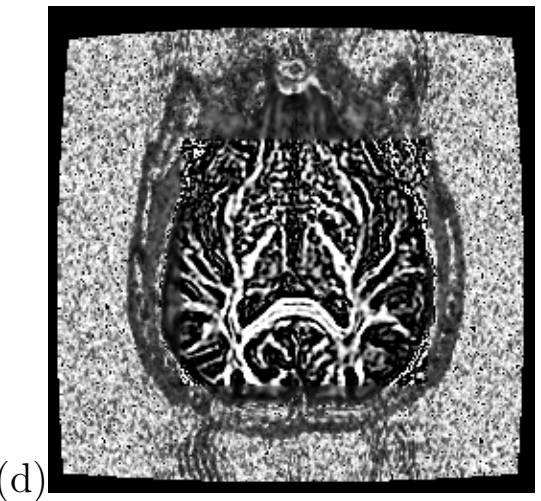
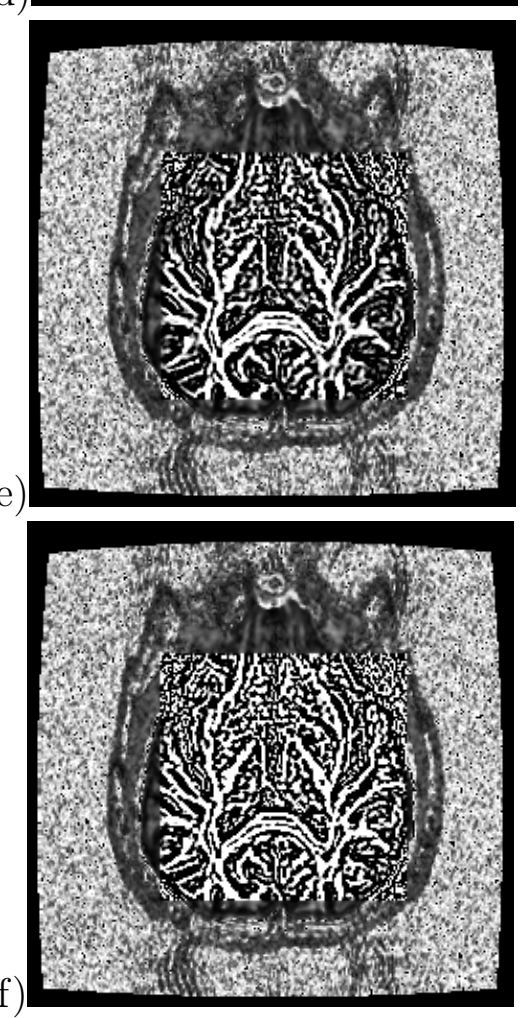

Figure 5: Comparison of texture-segmentation performance between an original grey image and its fractional differential using FCD-2 with a $5 \times 5$ mask. (a) Original image, (b) 1.2 order FCD-2, (c) 1.4 order FCD-2, (d) 1.5 order FCD-2, (e) 1.6 order FCD-2, (f) 1.8 order FCD-2. 
simple sensitivity study to obtain a reasonable trade off between precision and visual effect. In this work we only report the range of values considered. In future work we will perform a rigorous evaluation of how the fractional order affects the performance of our algorithm.

Acknowledgements: The authors gratefully acknowledge the help and interest in our work by Professor Kerrie Mengersen from QUT. Mr Yu also acknowledges the Centre for Complex Dynamic Systems Control for offering financial support for his PhD scholarship. We also thank the referees for their constructive comments and suggestions.

\section{References}

[1] R. C. Gonzalez, R. E. Woods, Digital Image Processing. Third Edition. Prentice Hall, New Jersey, USA, 2007. C591, C592

[2] C. S. Panda, S. Patnaik, Filtering corrupted image and edge detection in restored grayscale image using derivative filters, International Journal of Image Processing, 3(3):105-119, 2009.

http://www. cscjournals .org/csc/manuscript/Journals/IJIP/ volume3/Issue3/IJIP-28.pdf C592, C599

[3] Y. Zhang, Y. Pu, J. Zhou, Construction of Fractional differential Masks Based on Riemann-Liouville Definition, Journal of Computational Information Systems, 6(10):3191-3199, 2010. http:

//www.jofcis.com/publishedpapers/2010_6_10_3191_3199.pdf C592, C594

[4] F. Liu, P. Zhuang, V. Anh, I. Turner, K. Burrage, Stability and convergence of the difference methods for the space-time fractional advection-diffusion equation, J. Appl. Math. Comput., 191:12-21, 2007. doi:10.1016/j.amc.2006.08.162 C592 
[5] Q. Yu, F. Liu, I. Turner and K. Burrage, Stability and convergence of an implicit numerical method for the space and time fractional Bloch-Torrey equation, Phil Trans R Soc A, 371(1990):20120150, 2013. doi:10.1098/rsta.2012.0150 C592, C593

[6] E. Sejdić, I. Djurović, L. Stanković, Fractional Fourier transform as a signal processing tool: An overview of recent developments, Signal Processing, 91(6):1351-1369, 2011. doi:10.1016/j.sigpro.2010.10.008 C592

[7] B. Pesquet-Popescu and J. L. Véhel, Stochastic fractal models for image processing, IEEE Signal Processing Magazine, 19(5):48-62, 2002. doi:10.1109/MSP.2002.1028352 C592

[8] B. Mathieu, P. Melchior, A. Oustaloup, Ch. Ceyral, Fractional differentiation for edge detection, Signal Processing, 83(11):2421-2432, 2003. doi:10.1016/S0165-1684(03)00194-4 C592

[9] C. B. Gao, J. L. Zhou, J. R. Hu, F. N. Lang, Edge detection of colour image based on quaternion fractional differential, IET Image Processing, 5(3):261-272, 2011. http://digital-library.theiet.org/content/ journals/10.1049/iet-ipr.2009.0409 C592

[10] C. B. Gao, J. L. Zhou, X. Q. Zheng, F. N. Lang, Image enhancement based on improved fractional differentiation, Journal of Computational Information Systems, 7(1):257-264, 2011.

http://www. jof cis.com/publishedpapers/2011_7_1_257_264.pdf C592

[11] Y. Pu, J. Zhou and X. Yuan, Fractional differential mask: A fractional differential-based approach for multiscale texture enhancement, IEEE Transactions on Image Processing, 19(2):491-511, 2010. doi:10.1109/TIP.2009.2035980 C592, C593, C594, C596

[12] M. D. Ortigueira, Riesz potential operators and inverses via fractional centred derivatives, International Journal of Mathematics and 
Mathematical Sciences, 2006:48391, 2006.

doi:10.1155/IJMMS/2006/48391 C593

\section{Author addresses}

1. Q. Yu, Discipline of Mathematical Sciences, Queensland University of Technology, Brisbane 4001, Australia.

2. F. Liu, Discipline of Mathematical Sciences, Queensland University of Technology, Brisbane 4001, Australia.

mailto:f.liu@qut.edu.au

3. I. Turner, Discipline of Mathematical Sciences, Queensland University of Technology, Brisbane 4001, Australia.

4. K. Burrage, Discipline of Mathematical Sciences, Queensland University of Technology, Brisbane 4001, Australia;

Department of Computer Science and OCISB, University of Oxford, OX1 3QD, UK

5. V. Vegh, Centre for Advanced Imaging, University of Queensland, Brisbane 4072, Australia. 